\title{
Performance Evaluation of AODVH: An Ad hoc Networking Scheme for Hybrid Nodes
}

\author{
Yasmin Jahir $^{1}$, Mohammed Atiquzzaman ${ }^{2}$, Hazem Refai ${ }^{1}$, Peter G. LoPresti ${ }^{3}$ \\ ${ }^{1}$ School of Electrical and Computer Engineering, University of Oklahoma, Norman, OK. \\ ${ }^{2}$ School of Computer Science, University of Oklahoma, Norman, OK. \\ ${ }^{3}$ Department of Electrical Engineering, The University of Tulsa, Tulsa, OK. \\ Email: $\{$ yasmin, atiq\}@ou.edu
}

\begin{abstract}
Mobile Ad hoc networks (MANETs) are composed of a collection of independent nodes usually connected by low bandwidth Radio Frequency $(R F)$ links which are challenging for transmitting video and high bandwidth applications for rescue workers in emergency situations. Earlier we proposed a routing protocol, called AODVH to use for high bandwidth communication for search and rescue operations in a disaster area which consists of hybrid nodes using Free Space Optical (FSO) and RF links, with FSO being the primary link and $R F$ as backup in the case of failure of "FSO only" paths. The objective of this paper is to evaluate the performance of AODVH using ns-2 simulations with varying network size and compare with three other Ad hoc routing protocols. We also implemented and tested AODVH in the laboratory using a testbed consisting of Linux based Ad hoc nodes. Results show that AODVH performs better in terms of packet loss, endto-end delay, overhead, packet delivery ratio, route discovery frequency and throughput when compared to with the other three protocols.
\end{abstract}

Keywords: Network mobility, performance analysis, mobility model, ns-2 simulation, computer networks.

\section{INTRODUCTION}

An Ad hoc network is characterized by a multihop, rapidly deployable temporary network consisting of a set of nodes that do not rely on any preexisting infrastructure support or base station [1] and usually communicate with each other using RF links.

On the other hand, a rapidly deployable, reliable and high bandwidth network is needed during natural or man made disasters as regular communication infrastructure breaks down [2]. During this state of emergency, a Disaster Area Wireless Network (DAWN) can be formed consisting of helium-filled balloons that are tethered to the ground. The balloons carry self configurable routers which form an Ad hoc network up in the sky and communicate among themselves using Free Space Optical (FSO) and Radio Frequency (RF) links. The network architecture is shown in Fig. 1.

This work was supported by National Science Foundation grant no. 0725801.

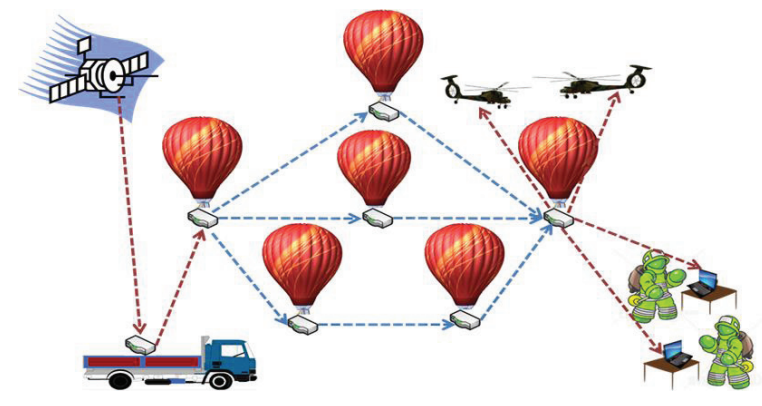

Fig. 1. Balloon wireless network of DAWN.

A Free Space Optical (FSO) link has significantly higher bandwidth and lower error rate [2] over RF links. However, link unavailability remains a big challenge for FSO links due to absorption (water vapor, carbon dioxide), scattering (fog) and shimmer (by light refraction, cloud cover, wind, etc.) [3]. Because of this intermittent characteristic of FSO links, it is not always possible to maintain " $F S O$ " links. As a solution, we developed a novel routing protocol called Ad hoc On-demand Distance Vector Hybrid (AODVH) in [4] which introduced hybrid nodes consisting of FSO and RF links, with RF links serving as backup in case of FSO link unavailability. AODVH [4] differs from other Ad hoc routing protocols (DSR [5], OLSR [6], AODV [7], AOMDV [8], AODVM [9], etc.) mainly in two aspects [4]:

- As previous unipath Ad hoc routing protocols require considerable time to find new routes in case of link failures, they are not suitable for DAWN.

- Earlier multipath protocols do not offer support for hybrid links consisting of FSO and RF.

The existing multipath Ad hoc routing algorithms [1], [8], [9] are extensions of Ad hoc reactive routing protocol viz. Dynamic Source Routing (DSR) [5] and Ad hoc On-demand Distance Vector (AODV) [7]. Lee et al. [1] developed a split multipath routing protocol with maximally disjoint paths for homogeneous RF nodes. Marina et al. [8] developed a loop free and link-disjoint multipath routing protocol (AOMDV). Zhenqiang et al. [9] proposed AODVM to discover multiple node-disjoint paths to achieve reliability in path setup for 
homogeneous RF nodes.

It is to be noted that all of the above [1], [8], [9], [10], [11] are based on homogeneous nodes and are not suitable for hybrid nodes.

There exists research works on Free Space Optics in literature. Bilgi et al. [12] proposed a simulation model for pure FSO node structures with intermittent connectivity pattern. Yuksel et al. [13] proposed a new FSO node design that uses spherical surfaces covered with transceiver models to maintain optical links when the nodes are in relative motion. These research works only considered optical links and hence did not meet our requirements of heterogeneous/hybrid links.

The main contribution of this paper is to evaluate the performance of AODVH [4] by comparing with other Ad hoc routing protocols through simulation and experimental setup.

We have simulated AODVH in ns-2 [14] with varying network size and compared the results with AODV [7] and two multipath protocols (AOMDV [8] and AODVM [9]). We also present the experimental implementation of our proposed protocol AODVH using four laptops and compared the results with AODVM [9].

The rest of the paper is organized as follows. We review our proposed protocol AODVH in Sec. II. The ns-2 [14] simulation setup is given in Sec. III, followed by the performance evaluation of simulation of AODVH and its comparison with AODV, AODVM, and AOMDV in Sec. IV. Sec. V presents the experimental setup for AODVH and experimental evaluation of AODVH with a comparison with AODVM is given in Sec. VI. Finally, concluding remarks are presented in Sec. VII.

\section{AODVH: AD hoc On-DEMAND Distance Vector ROUTING FOR HYBRID NODES}

In this section, we briefly discuss our proposed protocol AODVH [4] which is based on AODV [7]. AODVH [4] attempts to establish paths consisting of "FSO only" links as primary paths and uses RF links as backups when FSO links go down.

For our protocol, we implement a hybrid node structure that supports two heterogeneous interfaces having RF and FSO characteristics. We opted for using multiple instances of the wireless channel at the node level and used the MAC features of ns-2 [14] to get the address of the interface the message arrives on. We can thus keep track of the interface used to send or receive information. The nodes set the flags for FSO and RF in their respective RREQ tables based on the interface the message has been received. During the first stage of RREQ message, the immediate neighbors receive RREQ from the source. The RREQ table for node 1 is shown in Fig. 2.

Nodes 2 and 3 also set up their respective RREQ tables the same way. During stage 2 of RREQ message, these intermediate nodes send the RREQ messages received from the source to their neighbors. In this way, the duplicate RREQ messages, containing information regarding the type of interface (FSO, $\mathrm{RF}$ or both) used along the path, reach the destination node.

Upon receiving RREQs from its neighbors, the destination node starts generating RREPs. A forward path is setup during

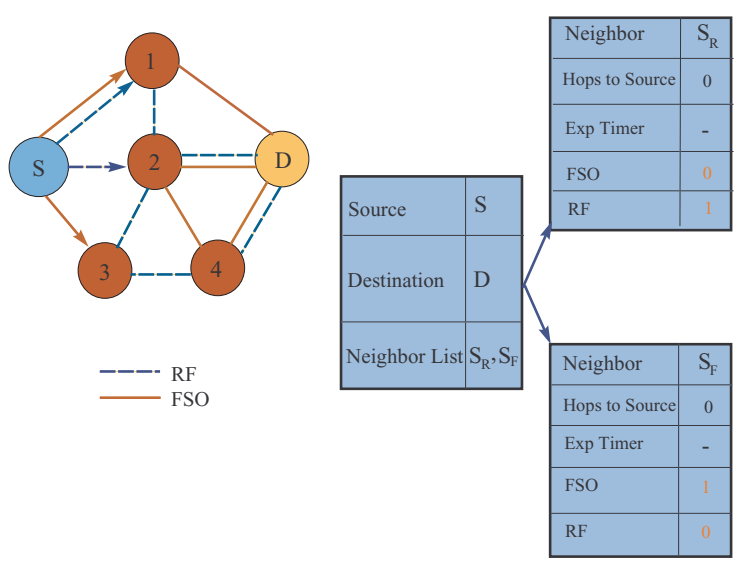

Fig. 2. Route Request (RREQ) Table for Node 1 (Stage 1).

the generation of the RREP message from source to the destination for data delivery. Due to the multipath nature of AODVH, the destination node replies to all RREQs it receives and tries to find "FSO only" paths first followed by "Hybrid paths" if "FSO only path" is not available. After forwarding the first RREP from node 1, the entry for node 1 is deleted from the RREQ tables of other intermediate nodes to achieve node disjointness. When all the RREPs reach the source $S$, we have multiple paths to send data from source to destination $\mathrm{D}$, with the first path being the primary one. The scenario is shown in Fig. 3.

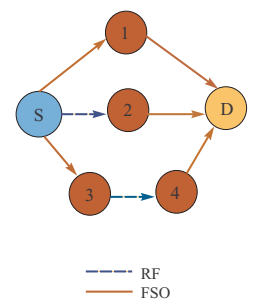

Fig. 3. Forward Path Set up from Source S.

The FSO link is directional (point to point). We adapted an FSO enhancement [12] for one of the interfaces in our hybrid node structure which used spherical multi transceiver structure to implement angular diversity and spatial reuse. Also this enhancement used an auto-alignment circuitry to handle the case of misalignment and drop out of FSO signals as described in [12].

\section{Simulation Setup}

We simulated our proposed protocol (AODVH) using Network Simulator (ns-2) [14]. Our objective is to evaluate the effectiveness of AODVH relative to AODV, AODVM and AOMDV, especially when route failures occur due to mobility 
with varying network size. Values of relevant simulation parameters for hybrid nodes with two interfaces are summarized in Table I.

TABLE I

SIMULATION PARAMETERS

\begin{tabular}{ll}
\hline Network Size & $1000 \mathrm{~m} \mathrm{x} 1000 \mathrm{~m}$ \\
Number of Nodes & $16,30,50$ and 80 \\
Simulation Time & $100 \mathrm{sec}$ \\
Traffic type & FTP \\
Channel Type1 & FSO Wireless Channel \\
Channel Type2 & Wireless Channel \\
Propagation Type1 & Free Space Optical \\
Propagation Type2 & Two Ray Ground \\
Channel capacity & $2 \mathrm{Mbps}$ \\
Node Transmission Range & $250 \mathrm{~m}$ \\
\hline
\end{tabular}

We vary the number of nodes with a fixed node speed of 10 $\mathrm{m} / \mathrm{s}$. We averaged the outcomes of five runs and generated the plots. Traffic pattern consists of FTP/TCP connection between a source and destination pair. The data packets have a fixed size of 1000 bytes in all the experiments. The maximum number of multipath routes was set to three, which has been shown to be an optimal number for multipath routing [8]. The random way point mobility model was used to simulate node movements [15].

\section{Simulation Results}

In this section, we evaluate the effectiveness of AODVH and compare with those of AODV, AODVM and AOMDV using packet loss, end-to-end delay, routing overhead and throughput as the performance metrics.

Packet Loss: We measure packet loss by the percentage of data packets that are dropped either at the source or at the intermediate nodes. We noticed that due to the increase in number of nodes the packet loss for all the protocols also increase due to link breaks with speed as shown in Fig. 4. For smaller node numbers (16), AODVH performs much better than other protocols (34\% less packet loss than AODV, and $19 \%$ less than AOMDV and AODVM). The packet loss for all the protocols increase with node number increase, but AODVH still drops fewer packets compared to other protocols as we used "FSO only" paths for AODVH which ensure faster and more reliable packet deliveries at the destination due to the multiple transceivers. In AODVH, AOMDV and AODVM, the source will have alternative routes to the destination; hence packet losses occur mostly at the intermediate nodes for these protocols. For AODV, this is not the case, as routes will fail more frequently with mobility and increased node number resulting in larger number of route discoveries from the source to find a route and higher packet loss.

End-to-End Delay: End-to-end delay is the sum of all possible delays encountered by a packet between a source and destination including data transmission, buffering during the route discovery period, delays at MAC layers, time of propagation, etc. Fig. 5 compares average end-to-end delay of AODVH with the other three routing protocols.

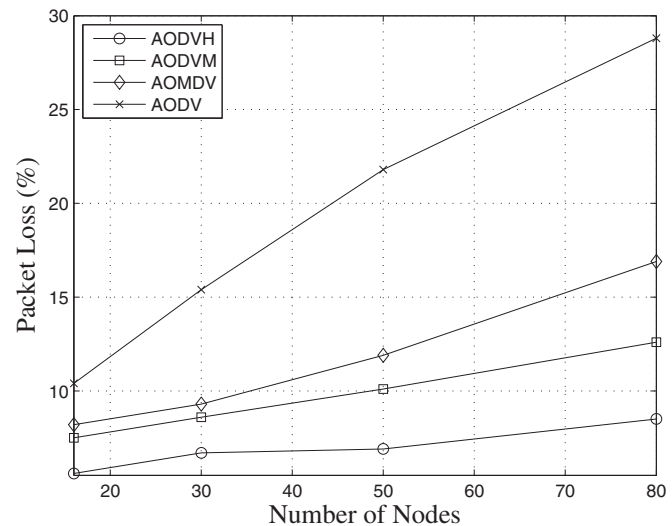

Fig. 4. Effect of number of nodes on packet loss.

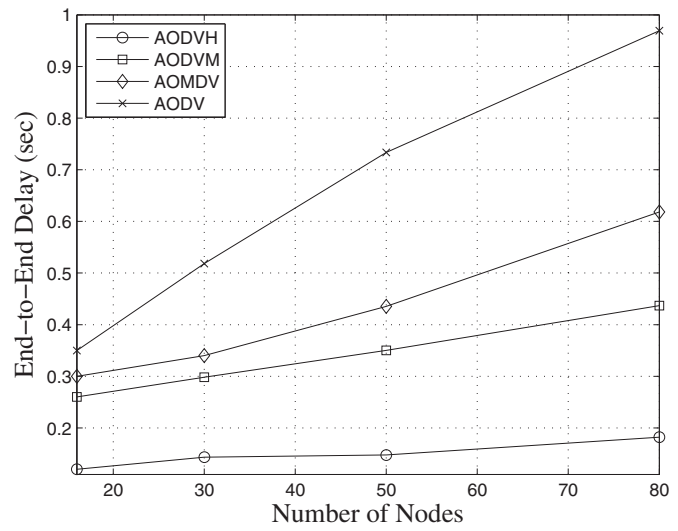

Fig. 5. Effect of number of nodes on end-to-end delay.

At a speed of $10 \mathrm{~m} / \mathrm{s}$, AODVH achieves minimum end-toend delay when compared to AODV, AOMDV and AODVM, respectively. "FSO only" paths result in decreased end-to-end delay because of the higher speed of the FSO links due to the multiple transceivers.

Routing Overhead: Routing overhead is measured by the total number of routing messages per second. Fig. 6 shows the routing overhead of AODVH, AODVM, AOMDV and AODV protocols as a function of node number. The routing overhead of AODVH is higher than AODVM as AODVH sends a great number of routing messages to create the double interface feature which is not required in AODVM. The routing overhead of AODV is the highest among the four protocols.

Throughput: We measure throughput by the total number of bits received at the destination per unit time. Fig. 7 compares the throughput of AODVH ("FSO and hybrid" paths) with AODVM (RF only paths) and AODV (RF only paths). We found that the throughput of AODVH is highest among the protocols due to the availability of multiple paths and higher bandwidth than other protocols using both the "FSO" link or 


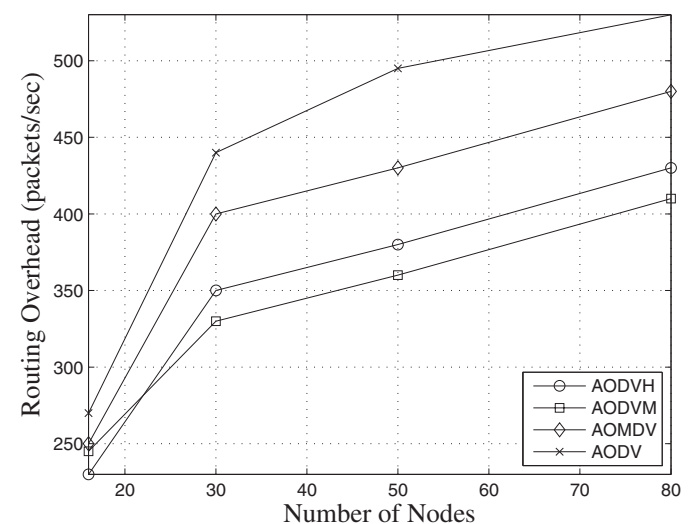

Fig. 6. Effect of number of nodes on routing overhead.

the "hybrid" link and the very minimal path pickup time in case of the breakage of primary path.

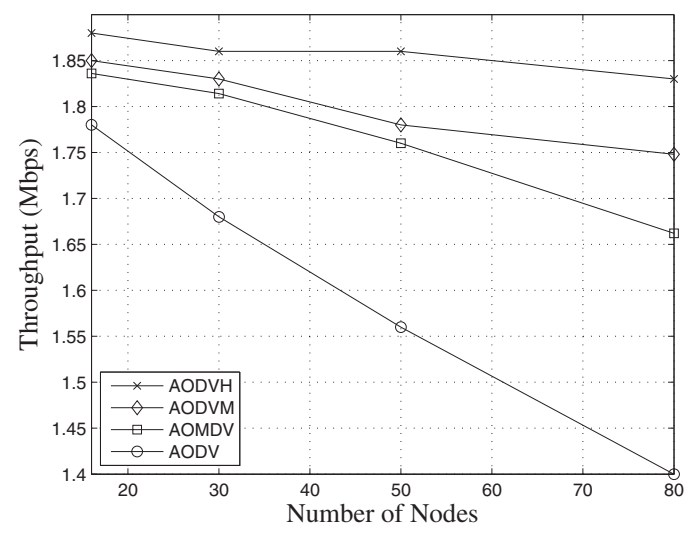

Fig. 7. Throughput vs. number of nodes.

AODVM and AOMDV perform better than AODV due to availability of alternate path in case of link failure of the primary path.

From the above results, we observe that AODVH achieves better performance than other three protocols (AODV, AOMDV and AODVM) in varying number of nodes in terms of packet loss, end-to-end delay and throughput.

\section{EXPERIMENTAL SETUP}

The design of the experimental implementation involves setting up AODVH on the machines and generating data traffic and observe the results gathered from the data captured at the destination node.

Description: The wireless cards were setup to run AODVH. We had two wireless cards setup on each machine to emulate the two interfaces on our proposed protocol. The first NIC (Network Interface Card) that we used is a PCMCIA card by AVAYA and the second one is the built in one in the machines with UBUNTU operating system. We implemented a third party version of AODVM, called AODV-UU [16] as shown in Fig. 8 and changed it to our proposed AODVH [4]. We used MAC filtering to implement the multi hop connection among the laptops.

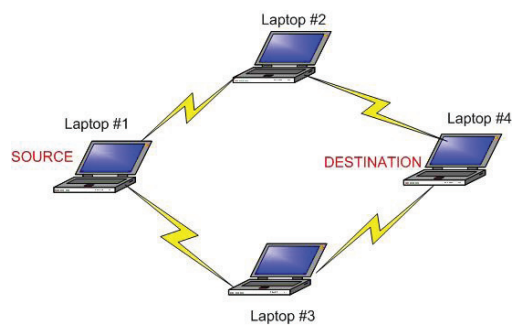

Fig. 8. Ad hoc multihop network using AODVM.

Our intention is to have higher data communication rate in one of these two interfaces to emulate the FSO network in AODVH. The Dell Mini Wireless Card was configured for 802.11g (up to $54 \mathrm{Mbps}$ ) and the AVAYA PCMCIA card was configured for $802.11 \mathrm{~b}$ (up to $11 \mathrm{Mbps}$ ). We used interface eth2 for higher data rate Dell Mini card and interface eth1 for lower data rate AVAYA PCMCIA card. We changed the AODV-UU [16] to adapt to use these two interfaces at the same time and use higher eth2 interface, if it is available for data communication. This higher bandwidth link will act as the "FSO link" for our proposed protocol AODVH, as described in Sec. II. This way we emulated the proposed AODVH to implement the protocol in real life Linux test beds to evaluate the protocol.

We used D-ITG (Distributed Internet Traffic Generator) [17], which is a platform capable of producing traffic at packet level and started single TCP flow with constant inter-departure time between packets and constant packet size.

The resulting flow from source to destination has the following characteristics:

1) 250 packets per second are sent (with constant interdeparture time between packets)

2) The size of each packet is equal to 1000 bytes

3) The run times of the experiment are 20,30,40 and 50 seconds respectively

We decoded the receiver log file on the destination host, which is laptop no. 4 which calculates the average values of bit rate, delay, throughput etc for specific run time.

\section{EXPERIMENTAL RESUlTS}

In this section we present numerical results showing the performance benefits of AODVH [4]. We evaluate the effectiveness of AODVH and compared with that of AODVM [9] in terms of packet loss, end-to-end delay and throughput using D-ITG [17] as described in Sec. V. We sent 1 Mbps TCP data from source node (laptop no. 1) to destination node (laptop no. 4) and averaged the outcomes of five runs for the data flow during a specific run time.

Packet Loss: Packet loss is measured as described in Sec. IV. Fig. 9 shows the packet loss of AODVH and AODVM. Due to 
high bandwidth communication link in AODVH, this protocol performs significantly better than lower bandwidth AODVM protocol. When we ran the data flow for 20 seconds, we found out that AODVH achieves $60 \%$ reduction in packet loss than AODVM. During the 30 and 40 second run of data flow, the primary path was taken down for AODVH. We noticed a rise in packet loss in case of AODVH which is still much less than AODVM (50\% less for $30 \mathrm{sec}$ and $35 \%$ less for $40 \mathrm{sec}$ ). This is due to the fact that the hybrid path was chosen by the protocol for the remaining time. Again, the percentage of packet loss of AODVH became $43 \%$ less than that of AODVM when we ran the data flow for 50 seconds using a combination of paths (0-25 FSO, 25-33 hybrid and 33-50 FSO).

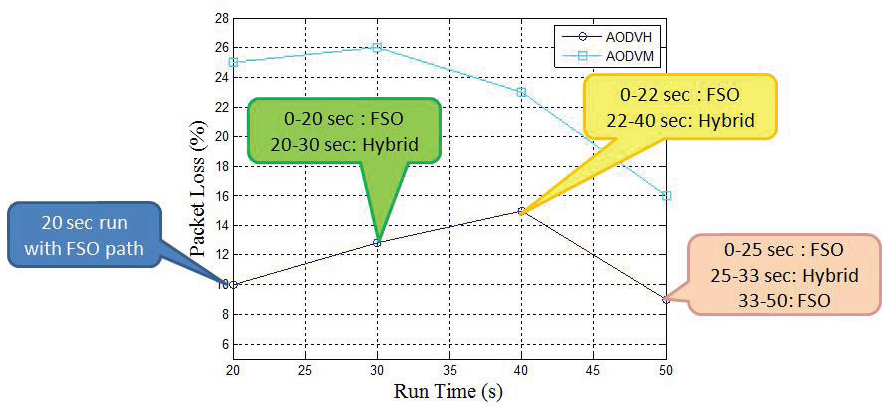

Fig. 9. Packet loss vs. run time in AODVH.

Throughput: We measure throughput as described in Sec. IV. Here we collect the number of bits at the destination laptop per second. Fig. 10 compares the throughput per second between AODVH and AODVM. The throughput of "FSO only" path of AODVH is higher (18\% more) than the throughput of AODVM. When we break the primary path of AODVH during 30 and 40 second runs, the throughput is reduced as AODVH used "Hybrid"/alternate paths. The reduced throughput is still higher than that of AODVM as this is the average throughput using both homogeneous "FSO" and "hybrid" paths.

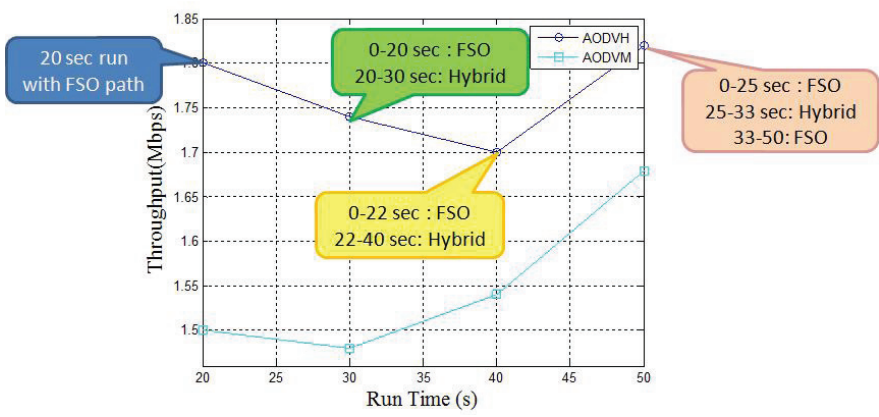

Fig. 10. Throughput vs. time in AODVH.

Problems/Limitations: The limitation of this experimental setup is that we could not implement a real FSO link as both of the interfaces of these machines use RF. Instead we made a difference between the two links in terms of data rate which is one important feature that distinguishes FSO from RF link. In real life, FSO link is very different than RF link and has other different characteristics as well.

\section{CONCLUSION}

In this paper, we evaluated AODVH, a multipath on-demand Ad hoc routing protocol for Disaster Area Wireless Network with simulation and experimental setup. Results validated that the multipath feature of AODVH significantly minimized packet loss, end-to-end delay, route discovery frequency when compared to AODV, AOMDV and AODVM. Throughput also improved while using hybrid paths when "FSO Only" path is unavailable. In addition to disaster recovery, DAWN can be very useful in military and exploration missions, home area wireless networking, networking intelligent devices, sensors, mobile robots, and on-the-fly conference applications.

\section{REFERENCES}

[1] S. J. Lee and M. Gerla, "Split multipath routing with maximally disjoint paths in ad hoc networks," in IEEE International Conference on Communications, June 11 - 14, 2001, pp. 3201-3205.

[2] P. Yan, J. J. S. Jr., H. H. Refai, and P. G. LoPresti, "Enhancing mobile ad hoc networks with free-space optics," Optical Engineering, vol. 46, no. 8, pp. 1-7, August 2007.

[3] S. Gurumani, H. Moradi, H. H. Refai, M. Atiquzzaman, and P. G. LoPresti, "Dynamic path reconfiguration among hybrid FSO/RF nodes," in GLOBECOM, New Orleans, LO, 30 Nov - 4 Dec 2008.

[4] Y. Jahir, M. Atiquzzaman, H. Refai, and P. G. LoPresti, "AODVH: Ad hoc On-demand Distance Vector Routing for Hybrid Nodes," in IEEE ICC, Cape Town, South Africa, May 23 - 27, 2010.

[5] D. Johnson, Y. Hu, and D. Maltz, "The Dynamic Source Routing Protocol (DSR) for Mobile Ad Hoc Networks for IPv4," RFC 4728, February 2007.

[6] T. Clausen and P. Jacquet, "Optimized Link State Routing Protocol," RFC 3626, October 2003.

[7] C. Perkins, E. Belding-Royer, and S. Das, "Ad hoc On-Demand Distance Vector routing," RFC 3561, July 2003.

[8] M. K. Marina and S. R. Das, "Ad hoc on-demand multipath distance vector routing," Wireless Communication and Mobile Computing, pp. 969-988, 2006

[9] Z. Ye, S. V. Krishnamurthy, and S. K. Tripathi, "A framework for reliable routing in mobile ad hoc networks," in IEEE INFOCOM, San Francisco, CA, 30 March - 3 April 2003, pp. 270-280.

[10] S. J. Lee and M. Gerla, "Aodv-br: backup routing in ad hoc networks," in Proceedings of IEEE Wireless Communications and Networking Conference (WCNC), Chicago, IL, September 23 - 28 2000, pp. 13111316.

[11] A. Valera, W. Seah, and S. Rao, "Cooperative packet caching and shortest multipath routing in mobile ad hoc networks," in Proceedings of IEEE INFOCOM, San Francisco, CA, April 1 - 3 2003, pp. 260-269.

[12] M. Bilgi and M. Yuksel, "Multi-element free-space-optical spherical structures with intermittent connectivity patterns," in INFOCOM, Phoenix, AZ, 13-18 April 2008, pp. 233-236.

[13] M. Yuksel, J. Akella, S. Kalyanaraman, and P. Dutta, "Free spaceoptical mobile ad-hoc networks: Auto-configurable building blocks," ACM/Springer Wirless Networks, vol. 15, no. 3, pp. 295-312, April 2009.

[14] K. Fall and K. Varadhan, "The ns manual," Ns Manual, 2002.

[15] J. Broch, D. Moltz, D. Johnson, Y.-C. Hu, and J. Jetcheva, "A performance comparison of multi-hop wireless ad hoc network routing protocols," in IEEE/ACM MobiCom, Dallas, Texas, 25-30 October 1998, pp. 85-97.

[16] J. Lorincz, N. Ukic, and D. Begusic, "Throughput comparison of aodvuu and dsr-uu protocol implementations in multi-hop static environments," in 9th International Conference on Telecommunications, Zagreb, Croatia, 13-15 June 2007, pp. 195-201.

[17] A. Botta, A. Dainotti, and A. Pescap, "Multi-protocol and multi-platform traffic generation and measurement," in INFOCOM 2007, DEMO Session, Anchorage (Alaska, USA), 6-12 May 2007. 